\title{
Encuesta a mujeres puérperas de autoeficacia $y$ actitud hacia la lactancia materna en un hospital de tercer nivel de atención
}

\author{
Survey in puerperal women on self-efficacy and attitude \\ of breastfeeding, from a tertiary care hospital
}

\begin{abstract}
Manuel Gil-Vargas, ${ }^{*+}$ Xitlalitl Dorantes-Vidal,* Magdalena León-López,* Sandra Maldonado-Castañeda*
* Servicio de Pediatría. Hospital General de Puebla “Dr. Eduardo Vázquez Navarro”. Puebla, México.
\end{abstract}

\begin{abstract}
RESUMEN
Introducción: En México, el porcentaje de lactancia materna (LM) en lactantes menores de seis meses bajó de 22.3 en 2006 a $14.5 \%$ en 2012. La actitud materna y la percepción para lograr una lactancia exitosa (autoeficacia) son factores que pueden ser modificables para mejorar la frecuencia de LM. Objetivo: Describir la autoeficacia y actitud de las madres hacia la LM en un hospital de ginecoobstetricia de tercer nivel de atención en México. Material y métodos: Encuesta descriptiva que se realizó a 331 puérperas, usando las escalas de autoeficacia Prenatal Breastfeeding Self-Efficiency Scale (PBSES) y de actitud, lowa Infant Feeding and Attitude Scale (IIFAS). Resultados: $88 \%$ presentó una actitud neutral y únicamente $10 \%$ tuvo una actitud positiva hacia la $L M$, mientras que con el instrumento PBSES se encontró que $94.9 \%$ tuvo una percepción de autoeficacia baja y $5.1 \%$ una precepción de autoeficacia alta. Conclusiones: Dados los resultados obtenidos parece necesario implementar acciones encaminadas a cambiar la actitud de las madres para mejorar la frecuencia y duración de la LM.
\end{abstract}

Palabras clave: Lactancia materna, puerperio, autoeficacia, actitud.

\begin{abstract}
Introduction: In Mexico, the percentage of breastfeeding (BF) in infants under six months decreased from 22.3 in 2006 to $14.5 \%$, in 2012. Maternal attitudes and the perception to achieve successful breastfeeding (self-efficacy) are factors that can improve of BF. Objective: To describe the selfefficacy and attitudes of mothers towards BF in a tertiary care hospital of Gynecology and Obstetrics, in Mexico. Material and methods: Descriptive survey applied to 331 puerperal women, using the Prenatal Breastfeeding Self-Efficiency Scale (PBSES) and lowa Infant Feeding and Attitude Scale (IIFAS). Results: Of the total, $88 \%$ presented a neutral attitude and only $10 \%$ had a positive attitude to BF. While with the PBSES instrument, it was found that $94.9 \%$ had a low self-efficacy perception and $5.1 \%$ had a high self-efficacy perception. Conclusions: Given the results obtained, it seems necessary to implement actions aimed at improving the attitudes of mothers, in order to increase the frequency and duration of $B F$.
\end{abstract}

Keywords: Breastfeeding, puerperium, self-efficacy, attitudes.

\section{WWVW medic}

\footnotetext{
+ Correspondencia: MGV, gilvm@yahoo.com

Conflicto de intereses: Los autores declaran que no tienen. Citar como: Gil-Vargas M, Dorantes-Vidal X, León-López M, Maldonado-Castañeda S. Encuesta a mujeres puérperas de autoeficacia y actitud hacia la lactancia materna en un hospital de tercer nivel de atención. Rev Mex Pediatr. 2020; 87(4): 132-136. https:// dx.doi.org/10.35366/95822
}

\section{INTRODUCCIÓN}

El conocimiento y las prácticas sobre lactancia materna (LM) es un tema primordial respecto a la atención del recién nacido (RN). De acuerdo con las pautas de alimentación, la LM siempre debe promoverse como la primera opción de alimentación, la cual debe iniciarse tan pronto como sea posible después 
del nacimiento, de preferencia dentro de la primera hora. Estas recomendaciones también señalan que la LM exclusiva debe continuarse en los primeros seis meses de vida. ${ }^{1}$

La disminución de la frecuencia de LM en México en los últimos años es evidente; según la Encuesta Nacional de Salud y Nutrición (ENSANUT 2012), el porcentaje de LM exclusiva en menores de seis meses se redujo entre 2006 y 2012 , de 22.3 a $14.5 \%$, siendo mayor en el medio rural, donde el descenso fue de $50 \%$ (de 36.9 a $18.5 \%) .^{2}$

La actitud hacia la LM es un indicador que puede predecir si las madres decidirán amamantar. La Escala Infantil de Alimentación y Actitud de Iowa (IIFAS, por sus siglas en inglés) es una escala autoaplicable que fue diseñada para medir la actitud de mujeres estadounidenses hacia la alimentación del lactante. Existe una versión en español que ya ha sido validada para población mexicana. ${ }^{3}$

Por otro lado, uno de los factores que afectan la LM es la autoeficacia, es decir, la percepción que las madres tienen de sí mismas sobre qué tan efectivo será amamantar. Se sabe que la autoeficacia está influenciada al menos por cuatro factores: habilidades desarrolladas previamente de la persona misma para la lactancia materna, experiencia al observar a otras mujeres que amamantan, apoyo verbal (de amigos cercanos, familiares, orientación sobre lactancia) y respuestas psicológicas (fatiga, estrés, ansiedad). ${ }^{4}$ La confianza forma parte del concepto de autoeficacia que constituye un aspecto psicológico que influye en la efectividad de un sujeto para afrontar un procedimiento estresante. ${ }^{5}$ La autoeficacia sobre la LM en mujeres embarazadas se puede medir con la Escala de Autoeficacia de la Lactancia Materna Prenatal (PBSES, por sus siglas en inglés), desarrollada en 2006 por Wells y su grupo, ${ }^{6}$ la cual es autoaplicable y ya se cuenta con una versión validada en español. ${ }^{7}$

El objetivo del presente estudio es describir la autoeficacia y actitud de las madres hacia la LM en un hospital de ginecoobstetricia de tercer nivel de atención en México.

\section{MATERIAL Y MÉTODOS}

Estudio descriptivo, transversal y prospectivo (encuesta descriptiva) que incluyó a 331 puérperas del Hospital de la Mujer Puebla, un hospital de tercer nivel de atención. Este estudio se realizó en los meses de junio y julio de 2019. Los criterios de inclusión fueron: puérperas que se encontraron en alojamiento conjunto, sin patología mamaria, con $\mathrm{RN}$ sanos a término. Se excluyó a mujeres a las que se les dificultara la LM y, cuando los RN presentaron alguna patología que les impidiera alimentarse.

Además de la solicitud de contestar los dos instrumentos en estudio se pidió a las madres información sobre edad, escolaridad, estado civil, lactancia previa y número de embarazos.

\section{Instrumentos}

La escala IIFAS utilizada consta de 17 elementos (Tabla 1) con una escala de Likert de 5, que van desde totalmente en desacuerdo hasta totalmente de acuerdo. Una actitud positiva hacia la LM se considera de 7085 puntos, actitud neutral de 49-69 puntos, mientras

Tabla 1: Elementos de la Escala Infantil de Alimentación y Actitud de lowa (IIFAS).

1. Los beneficios nutricionales de la leche materna duran sólo hasta que se le deja de dar

2. Alimentar con leche de fórmula es más adecuado que dar pecho

3. La lactancia materna favorece la unión madre-hijo

4. La lactancia materna es deficiente en hierro

5. Los bebés alimentados con fórmula son más propensos a ser sobrealimentados que los bebés alimentados con leche materna

6. La alimentación con leche en polvo es la mejor elección si la madre planea trabajar fuera del hogar

7. Madres que alimentan con leche de fórmula a sus bebés pierden una de las más grandes alegrías de la maternidad

8. Las madres no deberían dar pecho en lugares públicos como restaurantes

9. Los bebés alimentados con leche materna son más sanos que aquéllos alimentados por fórmula
10. Los bebés alimentados con leche materna son más propensos a ser sobrealimentados que los bebés alimentados con leche en polvo

11. Los papás se sienten excluidos si la madre da pecho a su bebé

12. La leche materna es el alimento ideal para los bebés

13. La leche materna es mucho más fácil de digerir que la leche de fórmula

14. La leche de fórmula es igual de saludable para un bebé que la leche de pecho

15. La leche materna es más adecuada que la leche de fórmula

16. La leche materna es más barata que la leche de fórmula

17. La mujer que ocasionalmente consume alcohol no debería dar pecho a su bebé 
Tabla 2: Escala de Autoeficacia de la Lactancia Materna Prenatal (PBSES).

1. Puedo hacer tiempo para la lactancia materna, incluso cuando me encuentro ocupada

2. Puedo dar pecho a mi bebé, incluso si estoy cansada

3. Puedo organizar mi día con base en alimentar a mi bebé con leche materna

4. Puedo darle pecho a mi bebé cuando estoy alterada

5. Puedo darle pecho a mi bebé, incluso si me causa alguna pequeña molestia

6. Puedo utilizar un extractor de leche para obtener leche

7. Puedo preparar la leche materna para que otros puedan alimentar a mi bebé

8. Puedo investigar qué necesito saber acerca de darle pecho a mi bebé

9. Puedo investigar la información que necesito sobre los problemas que tengo al darle pecho a mi bebé

10. Sé a quién preguntarle si tengo alguna pregunta sobre lactancia materna
11. Puedo hablarle a un asesor de lactancia si tengo problemas al darle pecho a mi bebé

12. Puedo hablar a mi médico sobre dar pecho a mi bebé

13. Puedo darle pecho a mi bebé cuando mi familia o amigos están conmigo

14. Puedo dar pecho a mi bebé alrededor de personas que no conozco

15. Puedo darle pecho a mi bebé cuando mi pareja está conmigo

16. Puedo darle pecho a mi bebé sin sentir vergüenza alguna

17. Puedo elegir dar pecho a mi bebé, incluso si mi pareja no quiere

18. Puedo elegir dar pecho a mi bebé, incluso si mi familia no quiere

19. Puedo hablar con mi pareja sobre la importancia de la lactancia materna

20. Puedo darle pecho a mi bebé por un año
Tabla 3: Características generales de las 331 mujeres encuestadas, de acuerdo al lugar de procedencia.

\begin{tabular}{|c|c|c|c|}
\hline & $\begin{array}{c}\text { Rural } \\
163(49.2) \\
n(\%)\end{array}$ & $\begin{array}{c}\text { Urbano } \\
168(50.7) \\
n(\%)\end{array}$ & $\begin{array}{l}\text { Total } \\
\text { n (\%) }\end{array}$ \\
\hline \multicolumn{4}{|l|}{ Edad en años } \\
\hline$\leq 18$ & 38 (23.3) & $27(16.1)$ & 65 (19.6) \\
\hline $19-30$ & 71 (43.5) & $84(50.0)$ & 155 (46.8) \\
\hline $31-40$ & $49(30.1)$ & 53 (31.5) & $102(30.8)$ \\
\hline$>40$ & $5(3.1)$ & $4(2.4)$ & $9(2.7)$ \\
\hline \multicolumn{4}{|l|}{ Escolaridad } \\
\hline Sin instrucción & $22(13.5)$ & $4(2.4)$ & $26(4.8)$ \\
\hline Primaria & $24(14.7)$ & $26(15.5)$ & $50(15.1)$ \\
\hline Secundaria & $31(19.0)$ & $37(22.0)$ & $68(20.5)$ \\
\hline Bachillerato & $50(30.7)$ & 53 (31.5) & $103(31.1)$ \\
\hline Licenciatura & $36(22.1)$ & 47 (27.9) & $83(25.1)$ \\
\hline Postgrado & 0 & $1(0.6)$ & $1(0.3)$ \\
\hline \multicolumn{4}{|l|}{ Estado civil } \\
\hline Casada & $42(25.8)$ & $50(29.8)$ & $92(27.8)$ \\
\hline Soltera & $13(7.9)$ & $20(11.9)$ & $33(9.9)$ \\
\hline Unión libre & 108 (66.3) & $97(57.7)$ & $205(61.9)$ \\
\hline $\begin{array}{l}\text { Divorciada/ } \\
\text { separada }\end{array}$ & 0 & $1(0.6)$ & $1(0.3)$ \\
\hline \multicolumn{4}{|l|}{ Lactancia previa } \\
\hline Sí & $88(53.9)$ & $83(49.4)$ & $171(51.7)$ \\
\hline \multicolumn{4}{|l|}{$\begin{array}{l}\text { Número de } \\
\text { embarazos }\end{array}$} \\
\hline 1 & $73(44.8)$ & 75 (44.6) & $148(44.7)$ \\
\hline 2 & $46(13.9)$ & 54 (32.1) & $100(30.2)$ \\
\hline 3 & 20 (12.3) & $25(14.9)$ & $45(13.6)$ \\
\hline 4 & $16(9.8)$ & $12(7.1)$ & $28(8.5)$ \\
\hline $5-7$ & $8(4.9)$ & $2(1.2)$ & $10(3.0)$ \\
\hline
\end{tabular}

que puntajes de 17-48 es una actitud negativa hacia la LM, por lo que es una actitud a favor dar fórmulas lácteas a los $\mathrm{RN}$.

Por su parte, la escala PBSES que se aplicó consta de 20 ítems (Tabla 2), con respuestas en escala tipo Likert de 5, que van de "no estoy segura" a "completamente segura". La puntuación total varía de 20 a 100, donde el mayor puntaje indica que la madre tiene una mejor percepción de que la lactancia materna será efectiva.

Análisis estadístico. Los datos se presentan solamente como estadística descriptiva de acuerdo a la escala de medición de las variables. Los datos se registraron y analizaron utilizando SPSS versión 24 .

Aspectos éticos. El protocolo de estudio fue aprobado por el comité de ética del propio hospital, y todas las participantes firmaron consentimiento informado.

\section{RESULTADOS}

Las características generales de las 331 mujeres se presentan en la Tabla 3. De ellas, $46 \%(\mathrm{n}=155)$ pertenecía al grupo de edad de 19 a 30 años, 31\% tenía como escolaridad el bachillerato, el estado civil más frecuente fue la unión libre (62\%), 52\% había dado LM previamente.

El puntaje promedio del IIFAS fue de 61.34 (DE 6.87), y el puntaje promedio para la escala PBSES fue de 79.20 (DE 13.73)

Al clasificar a las madres de acuerdo con el puntaje de la escala IIFAS se encontró que $2 \%$ tuvo una actitud 
Tabla 4: Clasificación de autoeficacia y actitud hacia la lactancia de acuerdo con IIFAS y PBSES.

\begin{tabular}{llcc} 
& & $n(\%)$ & Intervalo de confianza 95\% \\
\multirow{2}{*}{ IIFAS } & Actitud positiva hacia la lactancia materna & $33(10.0)$ & $7.1-13.6$ \\
& Actitud neutral & $290(87.6)$ & $83.6-90.8$ \\
\cline { 2 - 2 } & Actitud positiva hacia la fórmula láctea & $8(2.4)$ & $1.2-4.5$ \\
& Percepción de autoeficacia baja & $314(94.9)$ & $92.1-96.9$ \\
& Percepción de autoeficacia alta & $17(5.1)$ & $3.1-7.9$ \\
\hline
\end{tabular}

IIFAS = Escala Infantil de Alimentación y Actitud de lowa, PBSES = Escala de Autoeficacia de la Lactancia Materna Prenatal.

positiva hacia la fórmula láctea, $88 \%$ presentó una actitud neutral y únicamente $10 \%$ tuvo una actitud positiva hacia la LM (Tabla 4). En tanto que con el instrumento PBSES se encontró que 94.9\% tuvo una percepción de autoeficacia baja y $5.1 \%$ una precepción de autoeficacia alta (Tabla 4).

\section{DISCUSIÓN}

En México las normas oficiales mencionan que la atención que reciban las mujeres en edad reproductiva en cualquier unidad médica, debe contener un enfoque preventivo, educativo, de orientación y de consejería. ${ }^{8}$ Asimismo, se enfatiza sobre las ventajas de la LM, por lo que se señala la importancia de otorgar orientación sobre este tema durante el control prenatal. ${ }^{9}$

En nuestro estudio la mayoría de las puérperas tienen una actitud neutral y una percepción de autoeficacia baja frente a la LM, lo que hace suponer que las madres no amamantarán de manera efectiva, lo cual es congruente con los diferentes reportes que señalan que en México y otros países la frecuencia de LM ha disminuido. ${ }^{2}$ Uno de los factores que influyen en la menor tasa de lactancia es la falta de capacidad técnica para aconsejar y manejar las complicaciones y dificultades comunes por parte del personal de salud. ${ }^{10}$

La actitud que las futuras madres tengan sobre la posibilidad de amamantar, sin duda es un fuerte predictor del inicio de la LM, que la LM sea exclusiva y que se mantenga por tiempo prolongado. En este estudio encontramos que las participantes tienen una actitud neutral, lo cual es similar a otro estudio realizado en China. ${ }^{11}$ Esto nos hace reflexionar sobre la necesidad de evaluar la actitud hacia la lactancia durante el embarazo, a fin de implementar estrategias que fomenten una actitud positiva y favorezcan la lactancia materna.
Dentro de los factores que se han relacionado con tener una mejor adherencia a la práctica de LM, se encuentra mayor escolaridad, y el antecedente de haber amamantado previamente. ${ }^{11,12}$ Esto último supone que también habrá mejor percepción de autoeficacia.

Por último, con los resultados obtenidos parece importante incrementar la promoción, información y asesoramiento de la LM para tratar de generar más confianza en las mujeres y mejorar las tasas de LM.

\section{REFERENCIAS}

1. Shaw SC, Devgan A. Knowledge of breastfeeding practices in doctors and nurses: A questionnaire-based survey. Med J Armed Forces India. 2018; 74(3): 217-219.

2. Gutiérrez J, Rivera-Dommarco J, Shamah-Levy T, VillalpandoHernández S, Franco A, Cuevas-Nasu L et al. Encuesta Nacional de Salud y Nutrición 2012. Resultados Nacionales. [Internet]. Instituto Nacional de Salud Pública. 2012. p. 163-167. Disponible en: https://ensanut.insp.mx/encuestas/ensanut2012/doctos/ informes/ENSANUT2012ResultadosNacionales.pdf.

3. Aguilar-Navarro $\mathrm{H}$, Coronado-Castilleja A, Góme-Hernández O, Cobos-Aguilar H. Adaptación de la lowa Infant Feeding Attitude Scale en población mexicana. Acta Pediatr Mex. 2016; 37(3): 149-158.

4. Aydin A, Pasinlioglu T. Reliability and validity of a Turkish version of the prenatal breastfeeding self-efficacy scale. Midwifery. 2018; 64: 11-16.

5. Luszczynska A, Scholz U, Schwarzer R. The general self-efficacy scale: multicultural validation studies. J Psychol. 2005; 139(5): 439-457.

6. Wells K, Thompson N, Kloeblen-Tarver A. Development and psychometric testing of the prenatal breast-feeding self-efficacy scale. Am J Health Behav. 2006; 30(2): 177-187.

7. Juárez-Castelán MA, Rojas-Rusesell ME, Serrano-Alvarado K, Gómez-García JA, Huerta- Ibáñez A, Ramírez-Aguilar M. Diseño y validación de un instrumento para medir la autoeficacia para lactar de mujeres embarazadas mexicanas. Psychol Av Discip. 2018; 12(1): 25-34.

8. Hamze L, Jing M, Reifsnider E. Knowledge and attitudes towards breastfeeding practices: a cross-sectional survey of postnatal mothers in China. Midwifery. 2019; 74: 68-75.

9. Solano-Vergara BB, Hernández-Pérez AM, Maldonado-Vanegas S. Nivel de conocimientos sobre la lactancia materna en madres adolescentes en la ciudad de Valledupar, Cesar. 2019, pp. 55-64. 
10. Dennis CLE. Identifying predictors of breastfeeding self-efficacy in the immediate postpartum period. Res Nurs Health. 2006; 29: 256-268.

11. Blyth R, Creedy DK, Dennis C-LE, Moyle W, Pratt J. Effect of maternal confidence on breastfeeding duration: an application of breastfeeding self-efficacy theory. Birth. 2002; 29: 278-284.
12. Holbrook KE, White MC, Heyman MB, Wojcicki JM. Maternal sociodemographic characteristics and the use of the lowa Infant Attitude Feeding Scale to describe breastfeeding initiation and duration in a population of urban, Latina mothers: a prospective cohort study. Int Breastfeed J. 2013; 8 (7): 1-14. 